\title{
Analysis of Mathematical Model for Migration Law of Radon in Underground Multilayer Strata
}

\author{
Wei Zhang, ${ }^{1}$ Dongsheng Zhang, ${ }^{2,3}$ Xufeng Wang, ${ }^{2}$ Mengtang $\mathrm{Xu}^{2}$ and Hongzhi Wang ${ }^{2}$ \\ ${ }^{1}$ IoT/Perception Mine Research Center, National and Local Joint Engineering Laboratory of Internet Technology on Mine, \\ China University of Mining \& Technology, Xuzhou 221008, China \\ ${ }^{2}$ School of Mines, China University of Mining \& Technology, Xuzhou 221116, China \\ ${ }^{3}$ College of Geology \& Mining Engineering, Xinjiang University, Urumqi 830046, China
}

Correspondence should be addressed to Dongsheng Zhang; dshzhang123@126.com

Received 27 November 2013; Accepted 27 January 2014; Published 6 March 2014

Academic Editor: Jian Guo Zhou

Copyright (C) 2014 Wei Zhang et al. This is an open access article distributed under the Creative Commons Attribution License, which permits unrestricted use, distribution, and reproduction in any medium, provided the original work is properly cited.

This paper summarized the separation process of radon based on its geophysical-chemical properties. Taking into account the geological conditions of mining, the mathematical model of radon migration in underground multilayer strata (UMS) was established to investigate the distribution law of radon concentration in UMS. It was found that the distribution of radon concentration in UMS is affected by both the properties of the strata and the depth of cover and the radon concentration law varies at different depths even in the same layer stratum. At last, in order to validate the derivation result of the mathematical model of radon migration in UMS, the actual measured values (AMV) and the calculated values (CV) were compared further. As a result, the CV was found to be approximately equal to the AMV with deviation values (DV) less than 5\%, which indicates that the derivation result of the mathematical model of radon migration in UMS is correct.

\section{Introduction}

As a main energy resource in China, coal accounts for about $77 \%$ and $65 \%$ in primary energy production and consumption $[1,2]$, which plays an important role in nation's economic development. The result of a latest research conducted by the National Development and Reform Commission shows that the energy structures will not change greatly in the next fifty years [3]. Coal will not account for less than $60 \%$ in total energy consumption until 2015, and its ratio in primary energy consumption will reach up to $50 \%$ until 2050 (see Figure 1). In the past decades, the total coal production and consumption in China always ranked first in the world. However, coal capacity in China is still insufficient during this time [4], and its average annual increment is more than 0.2 billion tons (see Figure 2). In this situation, coal resources exploitation has become the precondition for economic development of China.

According to the latest results of coalfield prediction from China National Administration of Coal Geology [5], the natural overall distribution of coal resources presents north-poor-south-rich and west-more-east-less patterns in China. In recent years, the focus on coal resources exploitation has gradually shifted from east to west areas, which has formed two major mining areas of coal resources in the northwest represented by Inner Mongolia and the southwest represented by Guizhou [6]. However, the buried depths of northwest coalfields are shallow within 200 meters. They are located in the arid and semiarid areas, where the surface ecological environment systems are extremely sensitive [7, 8]. At present, large-scale and high-efficiency longwall mining has been widely used in western shallow coal seams. This has caused the mining-induced fractures to communicate with surface directly from bottom to top and led to a series of safety and environmental disasters including groundwater leakage, vegetation death, land desertification, and coal spontaneous combustion, which have made the potential ecological fragility into reality destruction $[9,10]$.

Therefore, the determination of spatiotemporal dynamic evolution law of the mining-induced fractures field in 


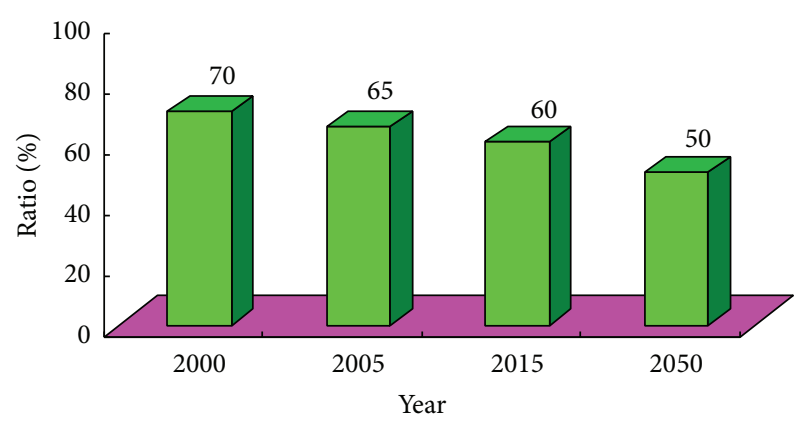

FIGURE 1: Coal ratio of primary energy consumption in China.

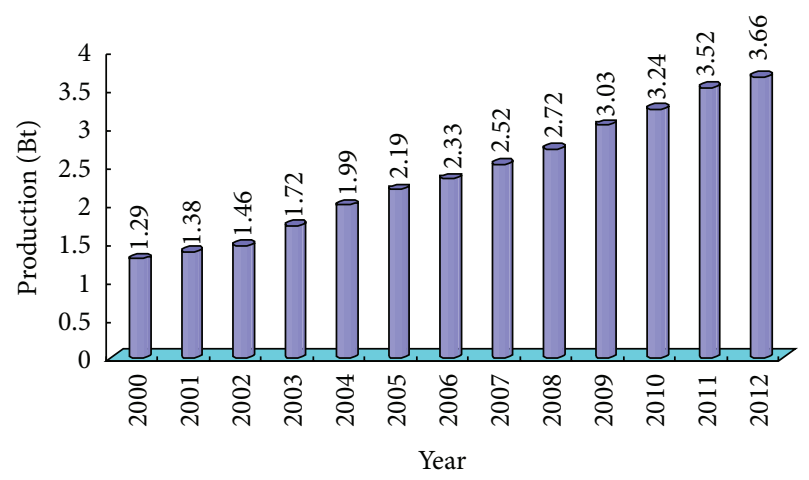

FIGURE 2: Coal production within thirteen years in China.

overlying strata and its relationship with underground water has been critical when it comes to the mitigation of mininginduced safety issues as well as the adverse environmental consequences in western mining areas in China. At present, there are many research methods for development characteristics of mining-induced fractures in overlying strata [1115]. However, the existing researches have some common shortcomings, such as heavy workload, complex operation, high cost, and difficulty to access data. Meanwhile, the effects and reliabilities are easy to be influenced by the underground geological conditions, by which it will be difficult to achieve the desired purposes. To solve these problems, geophysicalchemical properties of radon were applied in the field of mining engineering, and then radioactive measurement method was introduced to detect the dynamic evolution process of mining-induced fractures and its aquosity in overlying strata during underground coal mining [16-20]. During the process of radon detection on surface, the issues relative to radon cannot be ignored. The migration law of radon in UMS is the basis of radon detection on site, and the data of radon detection on surface is critical to the inverse analysis of the development characteristics of mining-induced fractures in overlying strata. Based on the inverse analysis, the corresponding control measures of mining techniques will be conducted for solving the three contradictions of coal mining and safety and environmental disasters in western mining areas, China. Hence, before carrying out the research work, the migration law of radon, especially in UMS, should be recognized, which is also the aim of this study.

\section{Geophysical-Chemical Properties of Radon and Its Separation Process}

2.1. Geophysical-Chemical Properties of Radon. Radon is a form of chemical element with a chemical symbol Rn and atomic number 86 . It is a zero group element of the sixth cycle in periodic table of chemical elements. In nature, radon has three kinds of common radioactive isotopes $\left({ }^{219} \mathrm{Rn},{ }^{220} \mathrm{Rn}\right.$, and ${ }^{222} \mathrm{Rn}$ ). In general, radon refers to ${ }^{222} \mathrm{Rn}$ with a halflife of 3.82 days. Radon molecule is a monatomic molecule, and its elemental form is usually gaseous, which is the only heaviest radioactive inert gas in contact with human. In the normal state, radon is colorless, tasteless, and odorless and is easily soluble in water and organic matters. The geophysicalchemical properties of radon are relatively stable, and it is difficult to produce chemical reactions with other substances [21]. As uranium $\left({ }^{238} \mathrm{U}\right)$ decays, it will eventually transform into radon. Since uranium exists in coal, rock, soil, and water with certain content in nature, radon is ubiquitous in natural environment.

In natural conditions, radon has strong migration ability, and it can migrate in geological environments by gaseous or dissolved form with ground water. Radon usually migrates by diffusion and convection effects in underground overlying strata, and the migration distance from underground strata to surface depends on different lithological characters. For example, the vertical migration distance in homogeneous sand is $360-420 \mathrm{~m}$ [22-24]. Moreover, the increased phenomenon of radon concentration in surface soil above coal spontaneous combustion area at $400 \mathrm{~m}$ depth had been observed in China [25]. These show that radon can migrate from underground overlying strata at $400-500 \mathrm{~m}$ depth to surface. Due to the radioactivity of radon, even if the concentration is very small, it can still be measured. Meanwhile, because of its geophysical-chemical properties of inert gas, it can migrate and accumulate in micropores or microfractures. Radon formed by radioactive decay in mining-induced fractures in underground overlying strata can penetrate the surface into air, which provides a basis for detecting radon on surface. For instance, German scholars found abnormal radon concentration in surface soil above the mining-induced area of deep shaft mining [26]. In this literature, the specific mining depth was not mentioned, but it can be deduced that the vertical migration distance is more than $400 \mathrm{~m}$.

2.2. Separation Process of Radon. Separation process of radon means that radon migrates from underground strata to surface and then spreads into the air. The whole separation process of radon can be divided into two stages of free radon generation and migration [27, 28] (see Figure 3). In the first stage, radium atom in media lattice of underground strata will decay into radon atom by emitting $\alpha$-particle, and the radon atom will escape from media lattice under nuclear recoil and emanation effects, then possibly enter into the interconnected microfractures, and thus generate the free radon. In the second stage, the free radon will migrate to the surface under diffusion and convection effects from 


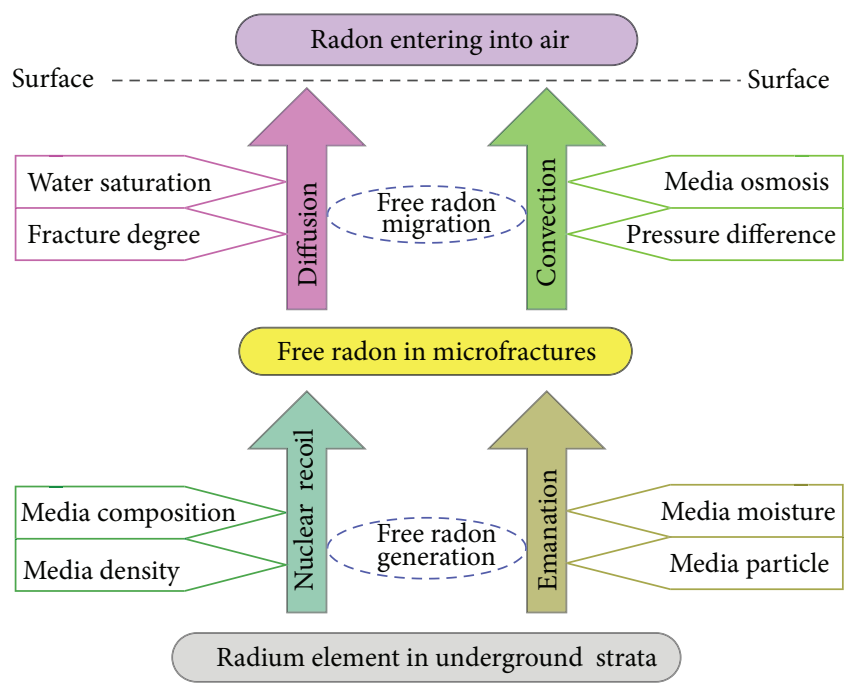

FIGURE 3: Separation process of radon.

the media microfractures and then will eventually escape from the surface into the air.

\section{Analysis of Mathematical Model for Migration Law of Radon}

It is known that the migration of chemical elements in porous media has been extensively studied over the past few years. For example, Srivastava and Jim Yeh developed a three-dimensional numerical model for the simulation of water flow and chemical transport through variably saturated porous media [29]. Diliunas et al. applied the thermodynamical calculation method and the WATEQ4 software to study the forms of chemical elements in water and their impact on migration [30]. Kitano et al. discussed the migration of chemical elements through phases of the atmosphere, hydrosphere, and lithosphere by dissolution experiments [31]. Ueno et al. investigated the vertical distribution patterns of the major and trace elements in the paddy soils derived from weathered volcanic ashes to estimate the relative mobilities of the elements in the paddy environment [32]. Savenko calculated the real water migration coefficients characterizing the mobility of chemical elements in the hypergenesis zone on the basis of basic salt components [33]. However, geochemical migration is an inextricable complex process that leads to the redistribution of chemical elements in subsurface. In this study, based on the migration law of radon in uniform porous media with arbitrary shape (UPMAS) and semiinfinite uniform porous media (SUPM), a mathematical model of radon migration in UMS has been established and the migration law of radon has been analyzed.

3.1. Analysis of Migration Law of Radon in UPMAS. Uniform porous media refer to media with uniform pores distribution, such as shapes, sizes, and properties [34]. Strictly speaking, there are no such ideal media in nature. Underground strata media vary from one mine site to another. Even if, at

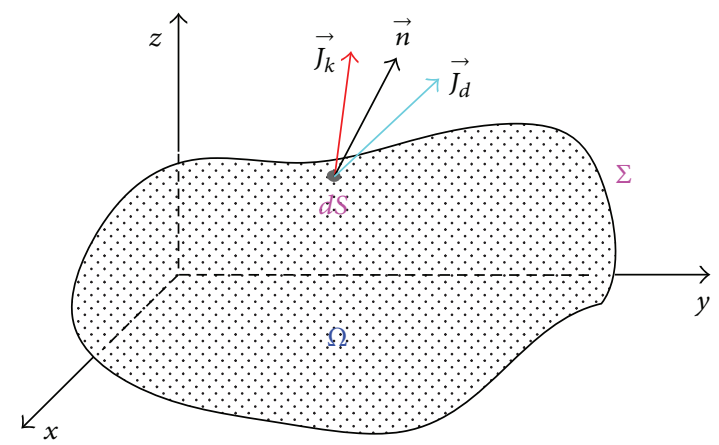

FIgURE 4: Mathematical model of radon migration in UPMAS.

different positions in the same layer stratum, the parameters of media density and porosity will also be different, this will lead to differences in radon concentration. Comparing to the volumes of the underground strata, their internal porosities and particle sizes are so small that they can be considered as uniform porous media approximately. Based on this hypothesis, the general differential equation of radon migration in UPMAS can be derived.

3.1.1. Mathematical Model Establishment of Radon Migration in UPMAS. It is hypothesized that there is a UPMAS; its whole closed volume is $\Omega$, and the entire closed surface area is $\Sigma$. Then, the unit vector $\vec{n}$ of surface element $\mathrm{d} S$ has been chosen as the outer normal direction, and the mathematical model of radon migration in UPMAS can be established (see Figure 4).

According to diffusion and convection effect, and radioactive decay laws, the change quantity of migratory radon in UPMAS with volume $\Omega$ should be equal to the generated migratory radon quantity minus the decayed radon quantity and the separated radon quantity in the unit time, which can be expressed as

$$
\begin{array}{r}
\frac{\partial}{\partial t} \iiint_{\Omega} C \eta \mathrm{d} v \\
=\iiint_{\Omega} A \mathrm{~d} v-\iiint_{\Omega} C \eta \lambda \mathrm{d} v-\oiint_{\Sigma} \overrightarrow{J_{k}} \mathrm{~d} S-\oiint_{\Sigma} \overrightarrow{J_{d}} \mathrm{~d} S \\
(C>0, \Omega>0, \Sigma>0),
\end{array}
$$

where $C$ is the radon concentration in micropores of media, $\mathrm{Bq} / \mathrm{m}^{3} ; \eta$ is the media porosity; $\lambda$ is the decay constant of radon/s; $\mathrm{d} v$ is the volume element of media; $A$ is the capacity of generating migratory radon in media per unit volume, $\mathrm{Bq} / \mathrm{m}^{3} \mathrm{~s} ; \overrightarrow{J_{d}}$ is the diffusion flux of radon through the closed surface with area $\Sigma, \mathrm{Bq} / \mathrm{m}^{2} s ; \overrightarrow{J_{k}}$ is the convection flux of radon through the closed surface with area $\Sigma, \mathrm{Bq} / \mathrm{m}^{2} \mathrm{~s}$.

According to the Gauss divergence theorem, the area integral form of closed surface can be transformed into 
volume integral form. Based on the $\overrightarrow{J_{k}}=-D \cdot \operatorname{grad} C$ and $\overrightarrow{J_{d}}=C \cdot \vec{v}$, two formulas can be obtained as

$$
\begin{gathered}
\oiint_{\Sigma} \overrightarrow{J_{k}} \mathrm{~d} S=-D \oiint_{\Sigma} \operatorname{grad} C \cdot \mathrm{d} S=-D \iiint_{\Omega} \operatorname{div}(\operatorname{grad} C) \mathrm{d} v \\
\oiint_{\Sigma} \overrightarrow{J_{d}} \mathrm{~d} S=\oiint_{\Sigma} C \cdot \vec{v} \mathrm{~d} S=\iiint_{\Omega} \operatorname{div}(C \cdot \vec{v}) \mathrm{d} v
\end{gathered}
$$

where $D$ is the diffusion coefficient of radon, $\mathrm{m}^{2} / \mathrm{s}$; vis the convection velocity of radon, $\mathrm{m} / \mathrm{s}$.

Substituting formula (2) into (1), formula (1) can be further written as

$$
\begin{aligned}
\frac{\partial}{\partial t} \iiint_{\Omega} C \eta \mathrm{d} v= & \iiint_{\Omega} A \mathrm{~d} v-\iiint_{\Omega} C \eta \lambda \mathrm{d} v \\
& +D \iiint_{\Omega} \operatorname{div}(\operatorname{grad} C) \mathrm{d} v \\
& -\iiint_{\Omega} \operatorname{div}(C \cdot \vec{v}) \mathrm{d} v .
\end{aligned}
$$

Formula (3) is a form of volume integral on media volume $\Omega$. Hence, differential calculation can be conducted on both sides of the equation. That is to say, formula (3) can be written as

$$
\eta \frac{\partial C}{\partial t}=A+D \operatorname{div}(\operatorname{grad} C)-\operatorname{div}(C \cdot \vec{v})-\lambda \eta C .
$$

It is clear that $\Delta=\partial^{2} / \partial x^{2}+\partial^{2} / \partial y^{2}+\partial^{2} / \partial z^{2}$ and $\nabla=$ $(\partial / \partial x) \vec{i}+(\partial / \partial y) \vec{j}+(\partial / \partial z) \vec{k}$. Hence, two formulas can be obtained as

$$
\begin{aligned}
D \operatorname{div}(\operatorname{grad} C) & =D \operatorname{div}\left(\frac{\partial C}{\partial x} \vec{i}+\frac{\partial C}{\partial y} \vec{j}+\frac{\partial C}{\partial z} \vec{k}\right)=D \Delta C, \\
\operatorname{div}(C \cdot \vec{v}) & =\operatorname{div}\left[C \cdot\left(\frac{\partial v}{\partial x} \vec{i}+\frac{\partial v}{\partial y} \vec{j}+\frac{\partial v}{\partial z} \vec{k}\right)\right] \\
& =\vec{v} \cdot \nabla C+C \cdot \nabla \cdot \vec{v} .
\end{aligned}
$$

Substituting formula (5) into (4), the general differential equation of radon migration in UPMAS can be expressed as

$$
\eta \frac{\partial C}{\partial t}=A+D \Delta C-\vec{v} \cdot \nabla C-C \cdot \nabla \cdot \vec{v}-\lambda \eta C \quad\left(\frac{\partial C}{\partial t}>0\right) .
$$

3.1.2. Radon Migration Equation in Steady State. Based on the definition of steady state, the change rate of radon concentration in emanation media is equal to zero with time in steady state; namely, $\partial C / \partial t=0$ [35]. Hence, the migration equation of radon in UPMAS in steady state can be written as

$$
A+D \Delta C-\vec{v} \cdot \nabla C-C \cdot \nabla \cdot \vec{v}-\lambda \eta C=0 \quad(C>0) .
$$

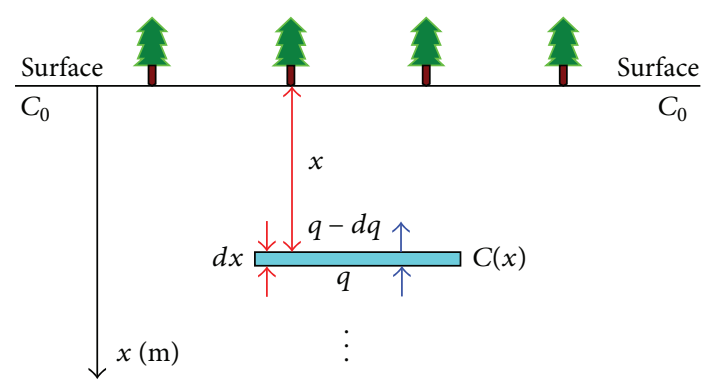

FIGURE 5: Mathematical model of radon migration in SUPM.

Particularly, one-dimensional condition is the most frequently used condition for radon migration. Hence, the onedimensional migration equation of radon in steady state in rectangular coordinate system can be expressed as

$$
\begin{aligned}
& A+D \frac{\mathrm{d}^{2} C}{\mathrm{~d} x^{2}}-v \frac{\mathrm{d} C}{\mathrm{~d} x}-\lambda \eta C=0 \\
& \left(v \text { is a constant, } \quad \frac{\mathrm{d} C}{\mathrm{~d} x}>0\right) .
\end{aligned}
$$

3.2. Analysis of Migration Law of Radon in SUPM. SUPM, such as the earth surface, can be defined as the uniform porous media with finite on one side and infinite on the other side. Radon migration in SUPM is a one-dimensional problem in steady state, which means that the radon concentration mainly depends on the depth of the location in the semiinfinite emanation media, while they are identical to each other as long as the depths are the same.

3.2.1. Mathematical Model Establishment of Radon Migration in SUPM. It is hypothesized that the earth is a SUPM; the $x$ one-dimensional coordinate along depth direction has been selected, and the mathematical model of radon migration in SUPM can be established (see Figure 5).

According to Figure 5, the change of radon quantity for any level thin layer $\mathrm{d} x$ of SUPM in unit time is mainly composed of three parts: the radon quantity difference in level thin layer caused by diffusion convection effects, which is $[(q-\mathrm{d} q)-q]=-\mathrm{d} q$; the radon quantity reduction in level thin layer due to radioactive decay of radon element; the radon quantity increase in level thin layer due to radioactive decay of radium element. Hence, the equation can be written as

$$
\begin{aligned}
\frac{\mathrm{d}}{\mathrm{d} t}[C(x) \eta \cdot S \mathrm{~d} x]= & {[(q-\mathrm{d} q)-q] } \\
& -C(x) \eta \lambda \cdot S \mathrm{~d} x+A \cdot S \mathrm{~d} x
\end{aligned}
$$

where $S$ is the area of any level thin layer $\mathrm{d} x, \mathrm{~m}^{2}$.

The radon quantity $q$ due to diffusion convection effects in the unit time can be represented as

$$
q=-D \frac{\mathrm{d} C(x)}{\mathrm{d} x} \cdot S+C \cdot v \cdot S
$$


Hence, $\mathrm{d} q$ can be represented as

$$
\mathrm{d} q=-D \frac{\mathrm{d}^{2} C(x)}{\mathrm{d} x^{2}} \cdot S \mathrm{~d} x+\frac{\mathrm{d} C(x)}{\mathrm{d} x} v \cdot S \mathrm{~d} x .
$$

Substituting formulas (10) and (11) into (9), formula (9) can be further expressed as

$$
\eta \frac{\mathrm{d} C(x)}{\mathrm{d} t}=D \frac{\mathrm{d}^{2} C(x)}{\mathrm{d} x^{2}}-v \frac{\mathrm{d} C(x)}{\mathrm{d} x}-C(x) \eta \lambda+A .
$$

It is known that the radon concentration will always be constant with time; namely, $\mathrm{d} C(x) / \mathrm{d} t=0$. Hence, formula (12) can be rewritten as

$$
\begin{array}{r}
D \frac{\mathrm{d}^{2} C(x)}{\mathrm{d} x^{2}}-v \frac{\mathrm{d} C(x)}{\mathrm{d} x}-C(x) \eta \lambda+A=0 \\
(x>0, C(x)>0) .
\end{array}
$$

Formula (13) can be simplified as

$$
\frac{\mathrm{d}^{2} C(x)}{\mathrm{d} x^{2}}-\frac{v}{D} \frac{\mathrm{d} C(x)}{\mathrm{d} x}-\frac{\eta \lambda}{D} C(x)=-\frac{A}{D} .
$$

The general solution of formula (14) can be derived as

$$
\begin{array}{r}
C(x)=C_{1} e^{\left[\left(v+\sqrt{v^{2}+4 D \eta \lambda}\right) / 2 D\right] x} \\
+C_{2} e^{\left[\left(v-\sqrt{v^{2}+4 D \eta \lambda}\right) / 2 D\right] x}+\frac{A}{\eta \lambda} \\
\quad(x>0, C(x)>0) .
\end{array}
$$

The integral constants of $C_{1}$ and $C_{2}$ in general solution can be determined by boundary conditions: (1) when $x=0$, $C(x)=C_{1}+C_{2}+A / \eta \lambda=C_{0}$; (2) when $x \rightarrow+\infty, C(x)$ has a limit value. Hence, $C_{1}$ and $C_{2}$ can be calculated as

$$
C_{1}=0, \quad C_{2}=C_{0}-\frac{A}{\eta \lambda} .
$$

Substituting formula (16) into (15), the distribution law of radon concentration in SUPM can be expressed as

$$
\begin{array}{r}
C(x)=\left(C_{0}-\frac{A}{\eta \lambda}\right) e^{\left[\left(v-\sqrt{v^{2}+4 D \eta \lambda}\right) / 2 D\right] x}+\frac{A}{\eta \lambda} \\
(x>0, \quad C(x)>0) .
\end{array}
$$

3.2.2. Analysis on Distribution Law of Radon Concentration in SUPM. Based on formula (17), it is observed that the distribution state of radon concentration in SUPM conforms to an exponential distribution law and relates to the radon concentration $C_{0}$ in media boundary. Whichever value the radon concentration $C_{0}$ takes, the radon concentration will always gradually tend towards a limit value along with the distance away from the surface boundary of media; that is,

$$
C_{\infty}=\lim _{x \rightarrow+\infty} C(x)=\left(C_{0}-\frac{A}{\eta \lambda}\right) e^{-\infty}+\frac{A}{\eta \lambda}=\frac{A}{\eta \lambda} .
$$

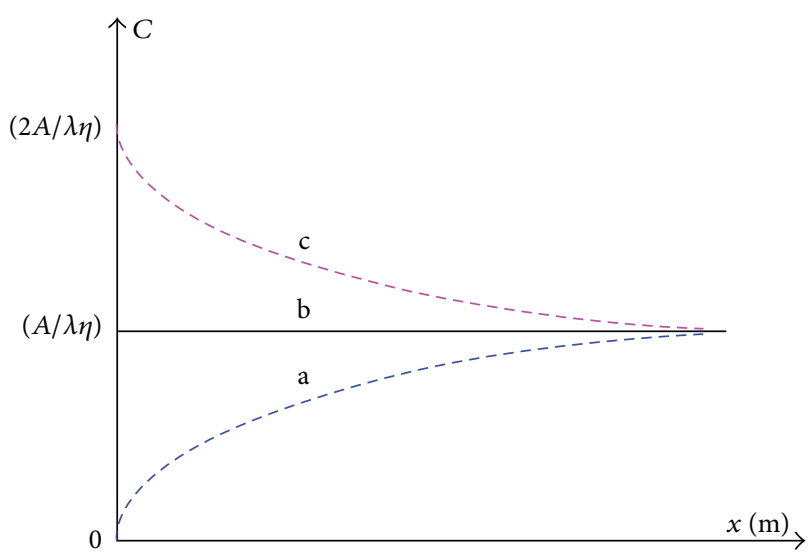
(a) $C_{0}=0$
(b) $C_{0}=A / \lambda \eta$
(c) $C_{0}=2 A / \lambda \eta$

FIGURE 6: Distribution state of radon concentration in SUPM with different $C_{0}$.

When the $C_{0}$ takes different values, the distribution state of radon concentration in SUPM can be shown in Figure 6.

3.3. Analysis of Migration Law of Radon in UMS. In the previous section of this paper. It is hypothesized that the earth is a semi-infinite medium and considered that all properties of medium under surface boundary are the same everywhere. As a matter of fact, the underground strata are composed of multilayer rocks with different lithologies in mining engineering field. They have their own properties, and they are not semi-infinite media. Hence, the migration law of radon is not entirely the same in the different layers. For this proposal, based on the mathematical model of radon migration in SUPM, the mathematical model of radon migration in UMS in accordance with geological conditions of mining has been established, and the migration law of radon in UMS has been analyzed.

\subsubsection{Mathematical Model Establishment of Radon Migration} in UMS. It is hypothesized that $p_{i}(i=1,2, \ldots, n)$ is a point in the $i$ th underground stratum medium and $x_{i}$ is the depth of $p_{i}$ relative to the top of the $i$ th underground stratum medium; the mathematical model of radon migration in UMS can be established (see Figure 7).

Based on the migration equation form of radon in SUPM in steady state, the general migration equation of radon in UMS in steady state can be analogized as

$$
\begin{array}{r}
D_{i} \frac{\mathrm{d}^{2} C_{i}\left(x_{i}\right)}{\mathrm{d} x_{i}^{2}}-v_{i} \frac{\mathrm{d} C_{i}\left(x_{i}\right)}{\mathrm{d} x_{i}}-C_{i}\left(x_{i}\right) \eta_{i} \lambda+A_{i}=0 \\
\left(i=1,2, \ldots, n, \quad x_{i}>0\right) .
\end{array}
$$




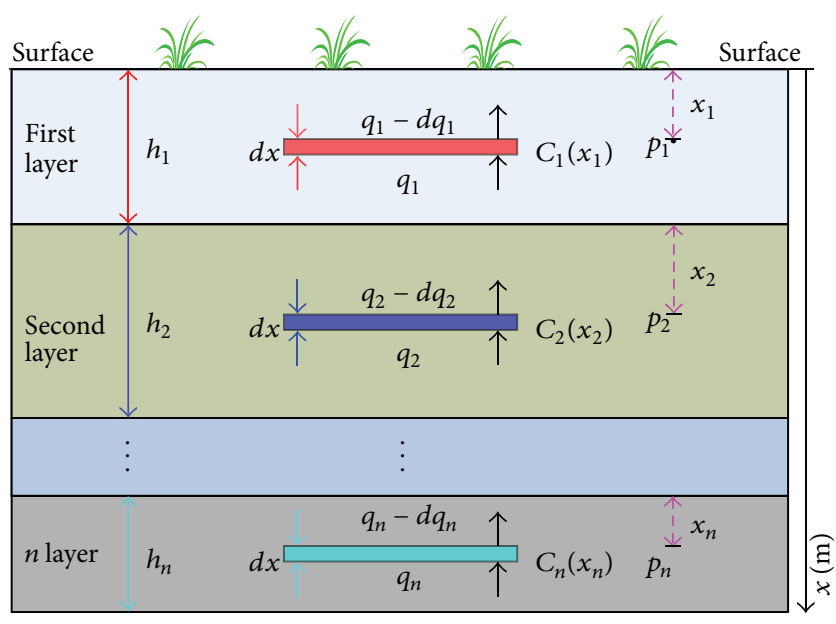

FIGURE 7: Mathematical model of radon migration in UMS.

According to the solution of second-order nonhomogeneous linear differential equation in higher mathematics, the general solution of formula (19) can be expressed as

$$
\begin{aligned}
& C_{i}\left(x_{i}\right)= m e^{\left[\left(v_{i}+\sqrt{v_{i}^{2}+4 D_{i} \eta_{i} \lambda}\right) / 2 D_{i}\right] x_{i}} \\
&+g e^{\left[\left(v_{i}-\sqrt{v_{i}^{2}+4 D_{i} \eta_{i} \lambda}\right) / 2 D_{i}\right] x_{i}}+\frac{A_{i}}{\eta_{i} \lambda} \\
& \quad\left(i=1,2, \ldots, n, \quad x_{i}>0\right) .
\end{aligned}
$$

Let $x$ be the depth relative to the earth surface; $x_{i}$ can be expressed as

$$
x_{i}=x-\sum_{j=0}^{i-1} h_{j} \quad\left(i \geq 2, h_{0}=0, x_{1}=x\right)
$$

Hence, formula (20) can be rewritten as

$$
\begin{aligned}
C_{i}(x)= & m e^{\left[\left(v_{i}+\sqrt{v_{i}^{2}+4 D_{i} \eta_{i} \lambda}\right) / 2 D_{i}\right]\left(x-\sum_{j=0}^{i-1} h_{j}\right)} \\
& +g e^{\left[\left(v_{i}-\sqrt{v_{i}^{2}+4 D_{i} \eta_{i} \lambda}\right) / 2 D_{i}\right]\left(x-\sum_{j=0}^{i-1} h_{j}\right)} \\
& +\frac{A_{i}}{\eta_{i} \lambda} \quad\left(i=1,2, \ldots, n, x_{i}>0\right) .
\end{aligned}
$$

The integral constants of $m$ and $g$ in the general solution can be determined by the boundary conditions: the radon concentration of two arbitrary contiguous layer strata is equal in parting position, because of the continuity of radon migration from the bottom to the top, and the change rate of radon concentration is also equal in parting position. Hence, the $k$ layer and $k+1$ layer can be selected to establish two equations and then determine the integral constant expressions of $m$ and $g$. According to formula (22), the radon concentration expressions of the $k$ layer and $k+1$ layer can be written as

$$
\begin{aligned}
C_{k}(x)= & m e^{\left[\left(v_{k}+\sqrt{v_{k}^{2}+4 D_{k} \eta_{k} \lambda}\right) / 2 D_{k}\right]\left(x-\sum_{j=0}^{k-1} h_{j}\right)} \\
& +g e^{\left[\left(v_{k}-\sqrt{v_{k}^{2}+4 D_{k} \eta_{k} \lambda}\right) / 2 D_{k}\right]\left(x-\sum_{j=0}^{k-1} h_{j}\right)}+\frac{A_{k}}{\eta_{k} \lambda}, \\
C_{k+1}(x)= & m e^{\left[\left(v_{k+1}+\sqrt{v_{k+1}^{2}+4 D_{k+1} \eta_{k+1} \lambda}\right) / 2 D_{k+1}\right]\left(x-\sum_{j=0}^{k} h_{j}\right)} \\
& +g e^{\left[\left(v_{k+1}-\sqrt{v_{k+1}^{2}+4 D_{k+1} \eta_{k+1}} \lambda\right) / 2 D_{k+1}\right]\left(x-\sum_{j=0}^{k} h_{j}\right)} \\
& +\frac{A_{k+1}}{\eta_{k+1} \lambda} .
\end{aligned}
$$

Based on the two boundary conditions, two equations of $C_{k}\left(\sum_{j=1}^{k} h_{j}\right)=C_{k+1}\left(\sum_{j=1}^{k} h_{j}\right)$ and $\mathrm{d} C_{k} /\left.\mathrm{d} x\right|_{\sum_{j=1}^{k} h_{j}}=$ $\mathrm{d} C_{k+1} /\left.\mathrm{d} x\right|_{\sum_{j=1}^{k} h_{j}}$ can be obtained. While $w_{k}=\left[\left(v_{k}+\right.\right.$ $\left.\left.\sqrt{v_{k}^{2}+4 D_{k} \eta_{k} \lambda}\right) / 2 D_{k}\right], p_{k}=\left[\left(v_{k}-\sqrt{v_{k}^{2}+4 D_{k} \eta_{k} \lambda}\right) / 2 D_{k}\right]$, and $L=A_{k+1} / \eta_{k+1} \lambda-A_{k} / \eta_{k} \lambda$, a matrix can be written as

$$
\left(\begin{array}{cc}
e^{w_{k} h_{k}}-1 & e^{p_{k} h_{k}}-1 \\
w_{k} e^{w_{k} h_{k}}-w_{k+1} & p_{k} e^{p_{k} h_{k}}-p_{k+1}
\end{array}\right)\left(\begin{array}{c}
m \\
g
\end{array}\right)=\left(\begin{array}{l}
L \\
0
\end{array}\right) .
$$

According to the Cramer rule in linear algebra, the integral constants of $m$ and $g$ can be calculated as

$$
\begin{aligned}
& m=\frac{(2+L) p_{k}-w_{k+1}-p_{k+1}}{\left(p_{k}-w_{k}\right) e^{w_{k} h_{k}}}, \\
& g=\frac{p_{k+1}+w_{k+1}-(2+L) w_{k}}{\left(p_{k}-w_{k}\right) e^{p_{k} h_{k}}} .
\end{aligned}
$$

Substituting formula (26) into (24), the distribution law of radon concentration in UMS can be expressed as

$$
\begin{aligned}
C_{i}(x) & \\
= & \frac{(2+L) p_{i}-w_{i+1}-p_{i+1}}{\left(p_{i}-w_{i}\right) e^{w_{i} h_{i}}} e^{\left[\left(v_{i}+\sqrt{v_{i}^{2}+4 D_{i} \eta_{i} \lambda}\right) / 2 D_{i}\right]\left(x-\sum_{j=0}^{i-1} h_{j}\right)} \\
& +\frac{p_{i+1}+w_{i+1}-(2+L) w_{i}}{\left(p_{i}-w_{i}\right) e^{p_{i} h_{i}}} e^{\left[\left(v_{i}-\sqrt{v_{i}^{2}+4 D_{i} \eta_{i} \lambda}\right) / 2 D_{i}\right]\left(x-\sum_{j=0}^{i-1} h_{j}\right)} \\
& +\frac{A_{i}}{\eta_{i} \lambda} \quad(i=1,2, \ldots, n, x>0),
\end{aligned}
$$

where $w_{i}=\left[\left(v_{i}+\sqrt{v_{i}^{2}+4 D_{i} \eta_{i} \lambda}\right) / 2 D_{i}\right] ; p_{i}=\left[\left(v_{i}-\right.\right.$ $\left.\left.\sqrt{v_{i}^{2}+4 D_{i} \eta_{i} \lambda}\right) / 2 D_{i}\right] ; L=A_{i+1} / \eta_{i+1} \lambda-A_{i} / \eta_{i} \lambda$.

3.3.2. Analysis of Distribution Law of Radon Concentration in UMS. Based on formula (27), it is observed that the distribution of radon concentration in UMS is affected by both the properties of the strata and the depth cover. The radon concentration is changed with depth even in the same layer stratum. In formula (27), $v_{i}, D_{i}, \eta_{i}$, and $A_{i}$ are all particular constants; they depend on their own media properties. 
TABLE 1: Parameters of specific property for the three-layer strata.

\begin{tabular}{lccc}
\hline Names & Symbols & Units & Values \\
\hline Convection velocity & $v_{i}(i=1,2,3)$ & $\mathrm{m} / \mathrm{s}$ & $5 \times 10^{-6} / 6 \times 10^{-6} / 4 \times 10^{-6}$ \\
Diffusion coefficient & $D_{i}(i=1,2,3)$ & $\mathrm{m}^{2} / \mathrm{s}$ & $5 \times 10^{-6} / 4 \times 10^{-6} / 3 \times 10^{-6}$ \\
Porosity & $\eta_{i}(i=1,2,3)$ & $/$ & $0.4 / 0.3 / 0.2$ \\
Capacity of generating migratory radon & $A_{i}(i=1,2,3)$ & $\mathrm{Bq} / \mathrm{m}^{3}$ & $3000 / 4000 / 5000$ \\
Decay constant of radon & $\lambda$ & $/ \mathrm{s}$ & $2.097 \times 10^{-6}$ \\
\hline
\end{tabular}

Meanwhile, the decay constant $\lambda$ is also a particular constant. Hence, so long as the specific values of parameters and depths have been known, the radon concentration values in UMS can be calculated. To validate the derivation result of the aforementioned mathematical model of radon migration in UMS, three-layer strata were selected to detect the radon concentration by KJD-2000R continuous emanometer at different depths. Then, the AMV and the CV were compared further. The parameters of specific property and size for the three-layer strata are shown in Table 1 and Figure 8.

Substituting the parameters in Table 1 and Figure 8 into formula (27), the radon concentration values of three different depths $(2 \mathrm{~m}, 5 \mathrm{~m}$, and $10 \mathrm{~m})$ in subsurface have been calculated. Meanwhile, the AMV has been detected. The two group values are shown in Figure 9. Based on the results, it is observed that the $\mathrm{CV}$ is approximately equal to the $\mathrm{AMV}$ with DV less than $5 \%$, which indicates that the derivation result of the mathematical model of radon migration in UMS is correct.

\section{Conclusions}

Separation process of radon can be divided into two stages of free radon generation and migration. In the first stage, the radium atom in media lattice of underground strata decays into radon atom by emitting $\alpha$-particle, and the radon atom escapes from media lattice into the interconnected microfractures under nuclear recoil and emanation effect, thus generating the free radon. In the second stage, under diffusion and convection effects, the free radon migrates to the surface and eventually escapes from the surface into the air.

The mathematical model of radon migration in UMS in accordance with geological conditions of mining has been established; the general migration equation of radon in UMS has been deduced, and the distribution law of radon concentration in UMS has been obtained. The calculation results indicate that the distribution of radon concentration in UMS is affected by both the properties of the strata and the depth of cover and the radon concentration law varies at different depths even in the same layer.

To validate the derivation result of the mathematical model of radon migration in UMS, three-layer strata were selected to detect the radon concentration by KJD-2000R continuous emanometer at different depths. The AMV and the $\mathrm{CV}$ were compared showing that the $\mathrm{CV}$ is approximately equal to the AMV with DV less than 5\%, which indicates

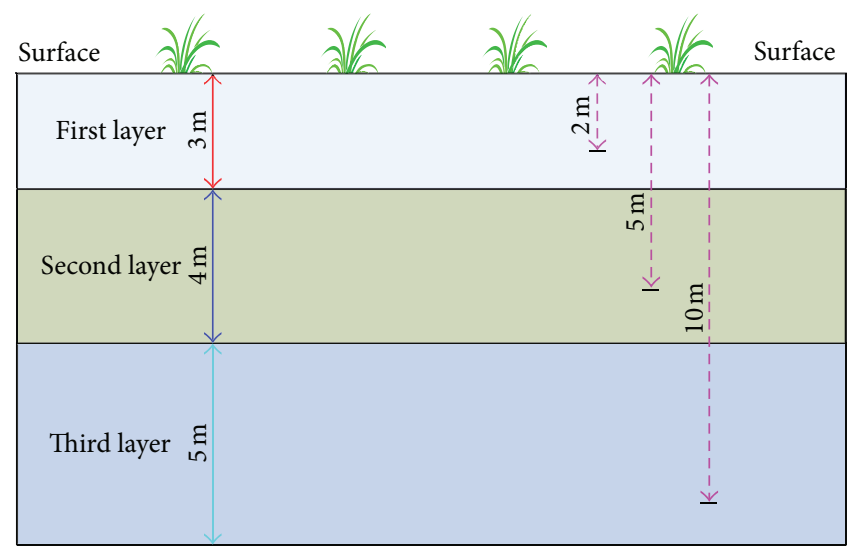

FIGURE 8: Size parameters of three-layer strata.

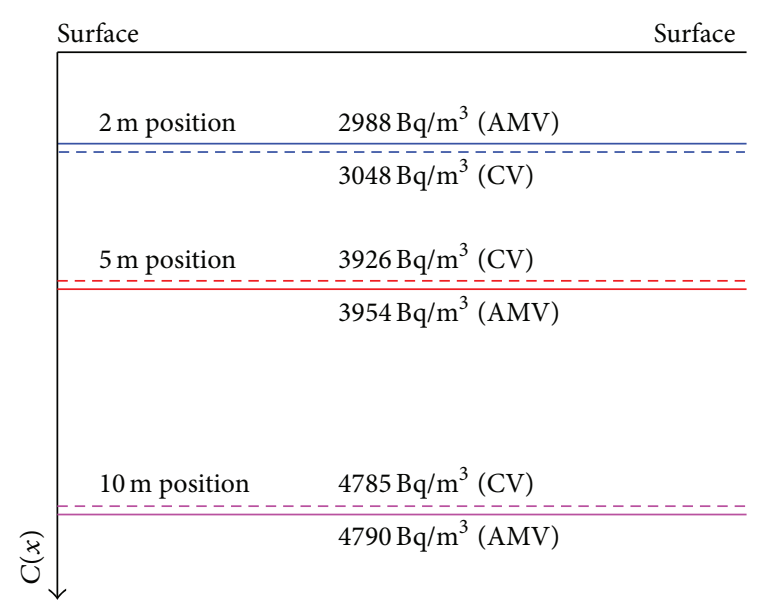

FIGURE 9: Radon concentration values at three different depths.

that the derivation result of the mathematical model of radon migration in UMS is correct.

\section{Conflict of Interests}

The authors declare that they have no financial and personal relationships with other people or organizations that can inappropriately influence their work, and they also declare that there is no conflict of interests regarding the publication of this paper. 


\section{Acknowledgments}

The research is financially supported by the Fundamental Research Funds for the Central Universities (no. 2013QNB24), Jiangsu Planned Projects for Postdoctoral Research Funds (no. 1302050B), the Sailing Plan of China University of Mining and Technology (no. 2012-05), and the National Natural Science Foundation of China (no. 51264035). The authors are grateful to Lecturer J. Q. Yu for his helpful advice. Special thanks were given to Doctor C. G. Zhang from the University of New South Wales, Australia, for language assistance. They also thank the academic editor Jian Guo Zhou and two anonymous reviewers for their constructive comments.

\section{References}

[1] S. Yu and Y.-M. Wei, "Prediction of China's coal productionenvironmental pollution based on a hybrid genetic algorithmsystem dynamics model," Energy Policy, vol. 42, pp. 521-529, 2012.

[2] J. Chang, D. Y. C. Leung, C. Z. Wu, and Z. H. Yuan, "A review on the energy production, consumption, and prospect of renewable energy in China," Renewable and Sustainable Energy Reviews, vol. 7, no. 5, pp. 453-468, 2003.

[3] B. Zhao, J. Xu, and J. Hao, "Impact of energy structure adjustment on air quality: a case study in Beijing, China," Frontiers of Environmental Science and Engineering in China, vol. 5, no. 3, pp. 378-390, 2011.

[4] H. M. Yu and H. Chen, "Production output pressure and coal mine fatality seasonal variations in China, 2002-2011," Journal of Safety Research, vol. 47, no. 1, pp. 39-46, 2013.

[5] J. H. Mao and H. L. Xu, Prediction and Evaluation of Coal Resources in China, Science Press, Beijing, China, 1999.

[6] D. Zhang, G. Fan, Y. Liu, and L. Ma, "Field trials of aquifer protection in longwall mining of shallow coal seams in China," International Journal of Rock Mechanics and Mining Sciences, vol. 47, no. 6, pp. 908-914, 2010.

[7] L.-Q. Ma, D.-S. Zhang, X. Li, G.-W. Fan, and Y.-F. Zhao, "Technology of groundwater reservoir construction in goafs of shallow coalfields," Mining Science and Technology, vol. 19, no. 6, pp. 730-735, 2009.

[8] Y. K. Yang, T. H. Kang, X. L. Hao, T. B. Zheng, and A. Wang, "Research on in-situ purification technique of mine water in Shendong mining area," Energy Education Science and Technology A, vol. 29, no. 1, pp. 209-216, 2012.

[9] D. Zhang, G. Fan, L. Ma, and X. Wang, "Aquifer protection during longwall mining of shallow coal seams: a case study in the Shendong Coalfield of China," International Journal of Coal Geology, vol. 86, no. 2-3, pp. 190-196, 2011.

[10] C.-M. Liu, G.-B. Fu, and L.-J. Li, "Water resources and ecological and environmental system construction in West China," Bulletin of Mineralogy Petrology and Geochemistry, vol. 21, no. 1, pp. 7-11, 2002.

[11] F. H. Ren, M. F. Cai, X. P. Lai, Y. H. Kang, and Z. G. Liu, "Monitoring and analysis of the damage height of overburden rock mass on the mined-out area," Journal of University of Science and Technology Beijing, vol. 26, no. 2, pp. 115-117, 2004 (Chinese).

[12] Y. Sun, Z. Xu, and Q. Dong, "Monitoring and simulation research on development of water flowing fractures for coal mining under Xiaolangdi Reservoir," Chinese Journal of Rock Mechanics and Engineering, vol. 28, no. 2, pp. 238-245, 2009 (Chinese).

[13] E. I. Shemyakin, G. L. Fisenko, M. V. Kurlenya et al., "Zonal disintegration of rocks around underground workings. Part 1: data of in situ observations," Soviet Mining Science, vol. 22, no. 3, pp. 157-168, 1986.

[14] C. F. Wu, S. D. Liu, S. L. Yang, T. Lu, and B. Wang, "Natural potential response during the coal rock failure process," Journal of China Coal Society, vol. 38, no. 1, pp. 50-54, 2013 (Chinese).

[15] T. Takano, T. Maeda, Y. Miki et al., "Detection of microwave emission due to rock fracture as a new tool for geophysics: a field test at a volcano in Miyake Island, Japan," Journal of Applied Geophysics, vol. 94, no. 7, pp. 1-14, 2013.

[16] W. Zhang, Mechanism research on detecting mining-induced fractures and its aquosity in overlying strata by radon on surface [Doctoral dissertation], China University of Mining and Technology, Xuzhou, China, 2012, 2012 Chinese.

[17] W. Zhang, D. S. Zhang, L. Q. Ma, X. F. Wang, and G. W. Fan, "Dynamic evolution characteristics of mining-induced fractures in overlying strata detected by radon," Nuclear Science and Techniques, vol. 22, no. 6, pp. 334-337, 2011.

[18] W. Zhang, D. Zhang, and G. Fan, "Design of comprehensive test system for detecting overlying strata mining-induced fractures on surface with radon gas," Mining Science and Technology, vol. 21, no. 6, pp. 823-827, 2011.

[19] W. Zhang, D. Zhang, L. Ma, X. Wang, G. Fan, and M. Xu, "Development of a comprehensive test system for detecting mining-induced fractures in overlying strata on surface with radon and its application," Chinese Journal of Rock Mechanics and Engineering, vol. 30, no. 12, pp. 2531-2539, 2011 (Chinese).

[20] Q. Bai, F. Fang, and X. Li, "Study of correlation of fracture caused by coal mine production and radon concentration," Computing Techniques for Geophysical and Geochemical Exploration, vol. 33, no. 2, pp. 175-178, 2011 (Chinese).

[21] W. Zhuo, T. Iida, J. Moriizumi, T. Aoyagi, and I. Takahashi, "Simulation of the concentrations and distributions of indoor radon and thoron," Radiation Protection Dosimetry, vol. 93, no. 4, pp. 357-368, 2001.

[22] L. Villalba, L. Colmenero Sujo, M. E. Montero Cabrera et al., "Radon concentrations in ground and drinking water in the state of Chihuahua, Mexico," Journal of Environmental Radioactivity, vol. 80, no. 2, pp. 139-151, 2005.

[23] A. Tricca, G. J. Wasserburg, D. Porcelli, and M. Baskaran, "The transport of $\mathrm{U}$ - and Th-series nuclides in a sandy unconfined aquifer," Geochimica et Cosmochimica Acta, vol. 65, no. 8, pp. 1187-1210, 2001.

[24] D.-G. Calugaru and J.-M. Crolet, "Identification of radon transfer velocity coefficient between liquid and gaseous phases," Comptes Rendus, vol. 330, no. 5, pp. 377-382, 2002.

[25] J. M. Wu and S. Q. Gao, "Study on temperature detection technique at fire district of coal spontaneous combustion and its application," China Safety Science Journal, vol. 14, pp. 109112, 2004 (Chinese).

[26] W. Rohnsch, S. Przyborowski, and E. Ettenhuber, "Investigation and evaluation of the radiation exposure situation in uranium mining areas of Eastern Germany," Radiation Protection Dosimetry, vol. 45, no. 1-4, pp. 127-132, 1992.

[27] L. L. Chyi, T. J. Quick, T. F. Yang, and C. H. Chen, “The experimental investigation of soil gas radon migration mechanisms and its implication in earthquake forecast," Geofluids, vol. 10, no. 4, pp. 556-563, 2010. 
[28] A. Varhegyi, J. Somlai, and Z. Sas, "Radon migration model for covering U mine and ore processing tailings," Romanian Journal of Physics, vol. 58, supplement, pp. 298-310, 2013.

[29] R. Srivastava and T.-C. Jim Yeh, "A three-dimensional numerical model for water flow and transport of chemically reactive solute through porous media under variably saturated conditions," Advances in Water Resources, vol. 15, no. 5, pp. 275-287, 1992.

[30] J. Diliunas, A. Jurevičius, and D. Karveliene, "Migration forms of main chemical elements in the groundwater of the Quaternary deposits of Lithuania," Baltica, vol. 22, no. 2, pp. 123-132, 2009.

[31] Y. Kitano, S. Kanamori, K. Kato et al., "Migration of chemical elements through phases of the atmosphere, hydrosphere and lithosphere in the Juneau Glacier area. I.," Geochemical Journal, vol. 22, no. 2-3, pp. 99-115, 1969.

[32] H. Ueno, M. Tsurumi, and M. Ichikuni, "Distribution and migration of chemical elements in paddy soil derived from Kanto loam," Chikyu Kagaku, vol. 26, no. 2, pp. 83-94, 1992.

[33] V. S. Savenko, "Water migration coefficients of chemical elements in the hypergenesis zone," Lithology and Mineral Resources, vol. 35, no. 4, pp. 345-350, 2000.

[34] A. J. Cooke and R. K. Rowe, "Extension of porosity and surface area models for uniform porous media," Journal of Environmental Engineering, vol. 125, no. 2, pp. 126-136, 1999.

[35] W. C. Ding, Y. Wang, Y. J. Li, F. Fang, and L. Yang, "A practical soil radon $\left({ }^{222} \mathrm{Rn}\right)$ measurement method," Nuclear Science and Techniques, vol. 21, no. 3, pp. 182-186, 2010. 


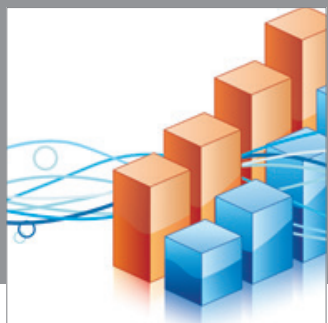

Advances in

Operations Research

mansans

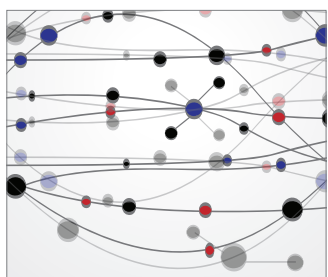

The Scientific World Journal
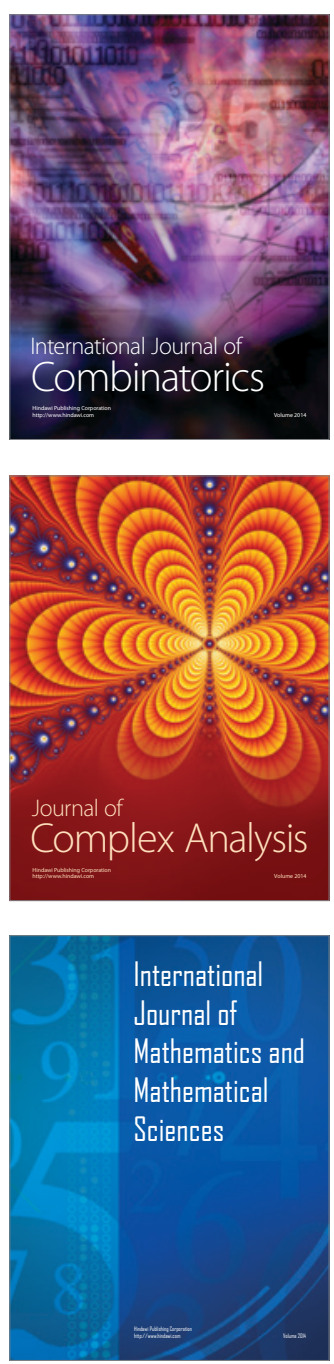
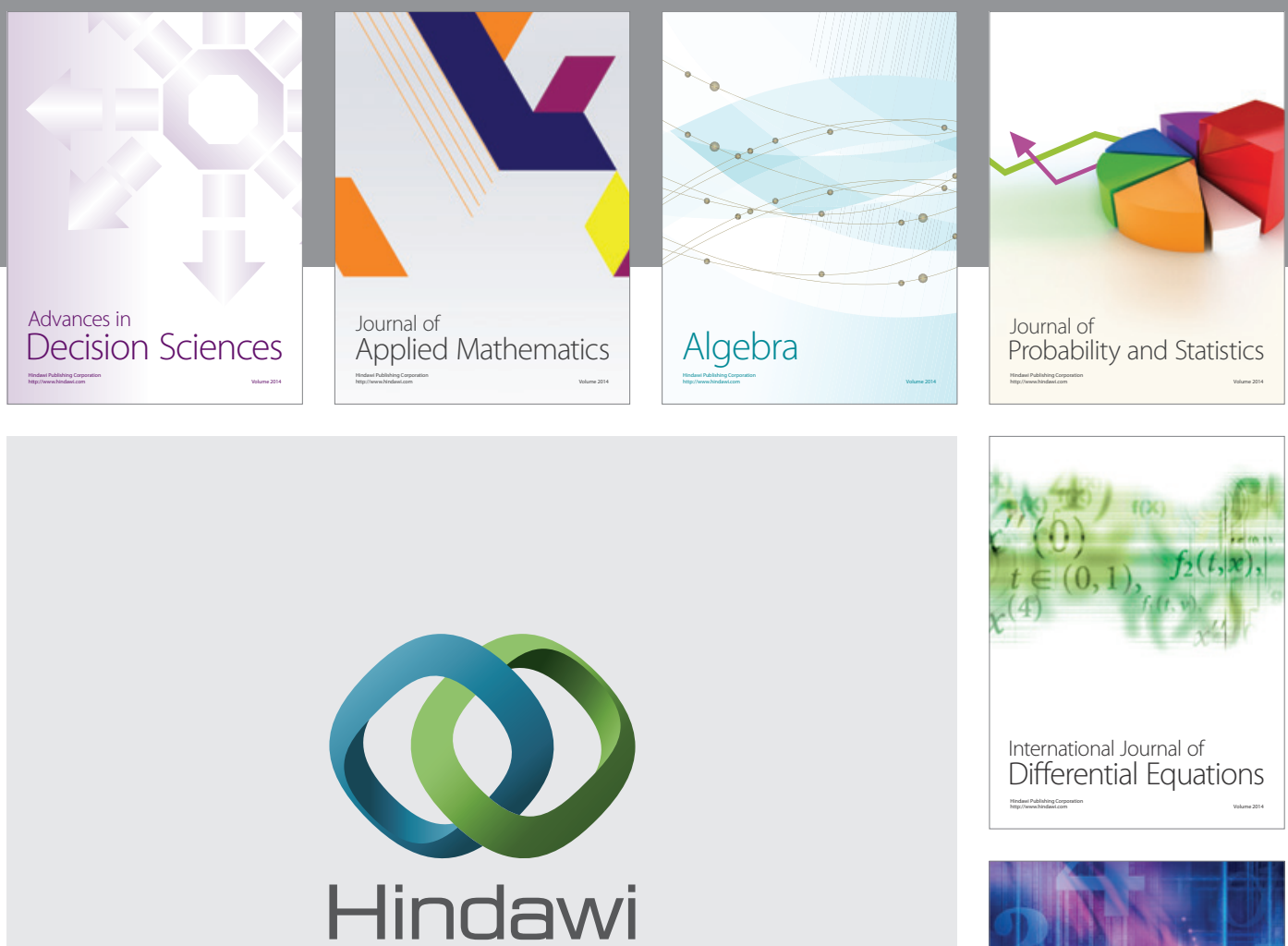

Submit your manuscripts at http://www.hindawi.com
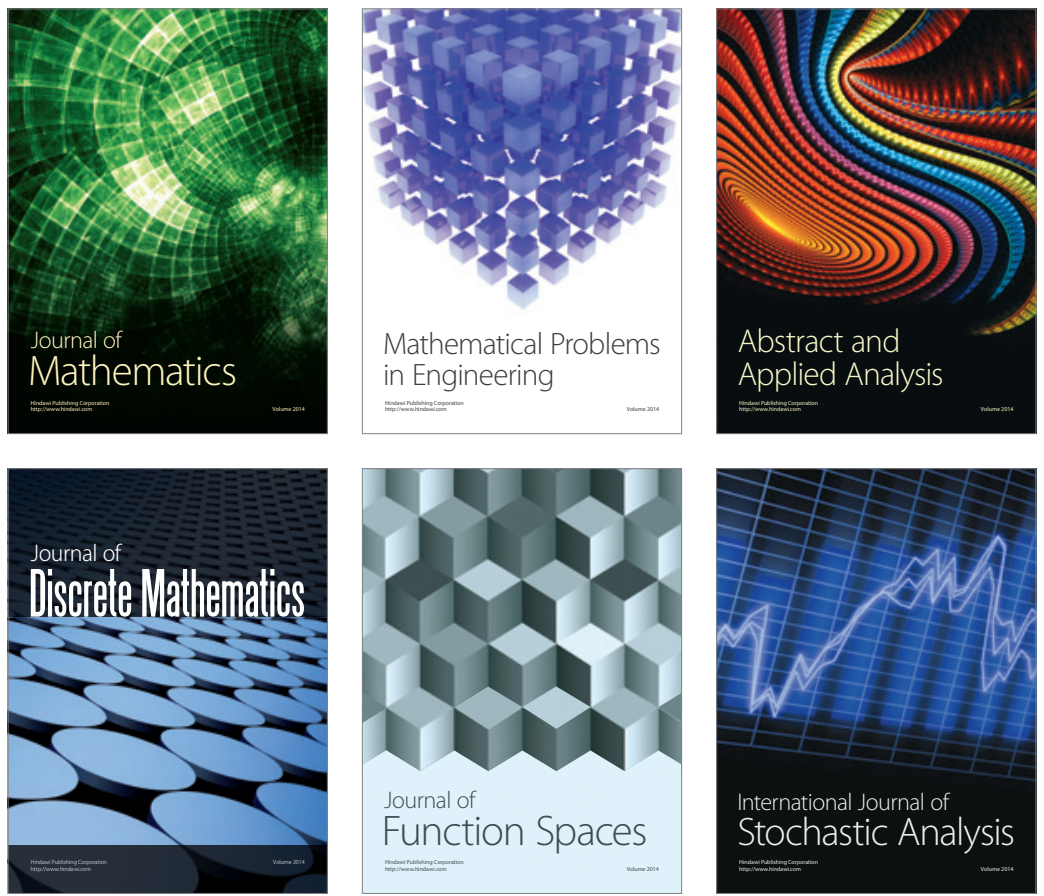

Journal of

Function Spaces

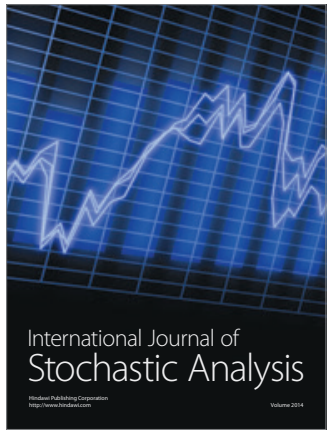

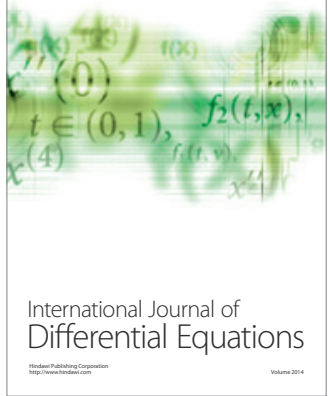
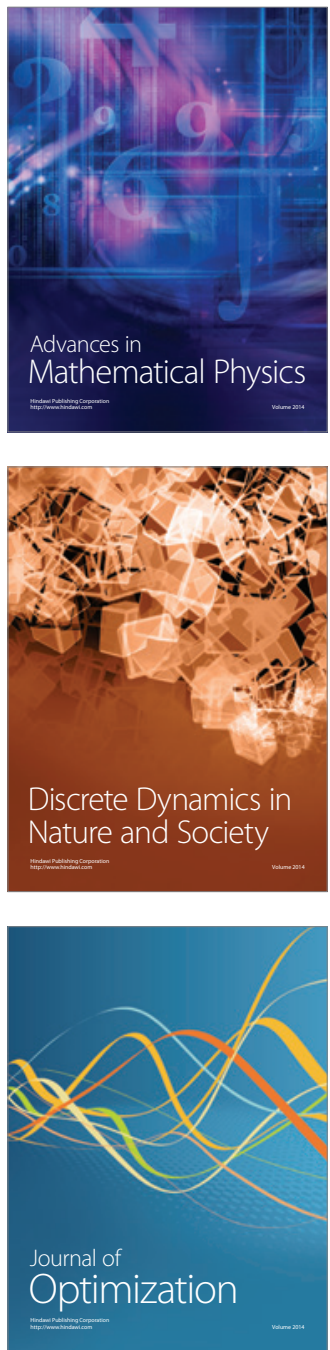